\title{
Cirugía por afecciones ováricas durante el embarazo. Experiencia institucional
}

\author{
María Teresa Peralta A*; Jorge Eduardo Caro**
}

RESUMEN: Vamos a presentar como objetivo general la experiencia institucional con un grupo de pacientes a quienes se les practicó laparotomía por afecciones ováricas durante la gestación. Específicamente se desea conocer la frecuencia, parámetros para diagnóstico, condiciones maternas al ingreso, indicaciones de la cirugía, preparación preoperatoria, momento de la misma, cuidados postoperatorios, anatomía patológica, evolución de la gestación y morbi-mortalidad materna y perinatal.

La investigación corresponde a un estudio descriptivo con una corte transversal y una sola medición en una muestra de la población, la cual está integrada por un universo de 6.500 pacientes atendidas en el Servicio de Alto Riesgo, desde el 10 . de enero de 1986 al 31 de octubre de 1993. Contamos con 18 gestantes sometidas a laparotomía lo cual representa una frecuencia de 2.7 por 1.000 , el $61.2 \%$ ingresan por dolor y en el 38.8\% el diagnóstico se hizo a través de hallazgo ecográfico. Se hace manejo expectante en gestaciones menores de 14 semanas, teniendo en cuenta un promedio de 18.8 semanas para el momento de la cirugía. Se utiliza anestesia peridural, no se presentan complicaciones intra o postoperatorias.

En el $\mathbf{8 8 . 8 \%}$ los resultados son compatibles con patología benigna, de los cuales el cistadenoma seroso es el más frecuente y en $11.2 \%$ se encontró neoplasia maligna con un cistadenocarcinoma seroso y un tumor de Krukenberg. Las gestaciones llegan en promedio a las 38.3 semanas y con parto espontáneo por vía vaginal en el $81.2 \%$. Recién nacidos en buenas condiciones y se reporta un caso de mortalidad materna y perinatal por carcinomatosis.

PALABRAS ClAVES: Afecciones ováricas, cirugía durante la gestación.

SUMMARY: We present our experience in a pregnant population, who underwent laparotomy due to ovarian entities. Frecuency, diagnostic criteria, maternal conditions at admission, indications of surgery, surgical preparation, timing, postsurgical care, pathology, pregnancy outcome and maternal and perinatal mortality and morbidity are presented.

The study, corresponds to a cross-sectional, descriptive analysis with a single measure in the universe considered. We reviewed 6500 patients treated in our High Risk Pregnancy service, from january 1986 to october 1993.18 patients underwent laparotomy, which represents a frecuency of 2.7 per $1000.61 .2 \%$, complained about pain and in $38.8 \%$, the diagnosis was made by ultrasound. Conservative management was considered for pregnancies under 14 weeks with an average of 18.8 at the time of surgery. Conductive epidural anesthesia was performed and no transoperative or postsurgical complications were found among patients. In $88.8 \%$ of cases, a bening lesion was reported, finding the serous cystadenoma the most frecuent, $11.2 \%$ of cases were reported as malignancies with a serous cystadenocarcinoma and a Krukenberg's tumor. An average of 38.3 weeks gestation was reached by pregnancies in the sample, with a vaginal delivery in $81.2 \%$ of cases. Newborns were found all in good conditions and one case of both maternal and fetal demise due to carcinomatosis is reported.

KEY WORDS: Surgery, pregnancy, ovarian entities.

\section{Introducción}

Los tumores ováricos son frecuentes durante el embarazo. La incidencia varía del 1 al $2 \%$. Los tumores malignos son raros, la incidencia varía desde 1 en 12.000 a 1 en 50.000 embarazos, con un promedio de 1 en 25.000. Los más frecuentes durante la gestación se relacionan con los teratomas quísticos benignos, seguido de los cistadeno-

\footnotetext{
* Profesor Asociado Departamento Ginecología y Obstetricia Universidad Nacional de Colombia. Coordinador Servicio Alto Riesgo.

** Residente de Tercer Año. Departamento de Ginecología y Obstetricia. Universidad Nacional de Colombia.
}

mas serosos, quistes simples, cistadenomas mucinosos, quistes pseudoováricos, quistes endometriales y cuerpos luteos entre otros.

Los problemas más importantes asociados con los tumores ováricos en el embarazo son el diagnóstico inicial y el diagnóstico diferencial. Sólo una tercera parte son asintomáticas y hallados durante el examen prenatal de rutina. El ultrasonido continúa siendo muy útil en el diagnóstico temprano de dichas lesiones. La sintomatología es muy variable, teniendo mayor frecuencia la presencia de dolor persistente, además de sintomatología urinaria, molestias gastrointestinales o molestias abdominales vagas. 
Los tumores mayores de $6 \mathrm{~cm}$ presentan aumento de complicaciones tales como torsión con hemorragia o ruptura; interferencia mecánica durante el trabajo de parto, infección y posibilidad de malignización, aunque es menos frecuente. Es decir que en general se está de acuerdo que masas mayores de $6 \mathrm{~cm}$ o masas sintomáticas de cualquier tamaño deben considerarse significativas y requieren atención.

En resumen en el tumor de ovario durante el embarazo se debe tener un alto índice de sospecha, se debe tener un diagnóstico temprano y se debe tratar sin dilación. La principal dificultad es el rechazo de la paciente a una exploración quirúrgica, sin embargo el peligro potencial para ella excede el daño para el feto. En general se deben manejar igual que en la mujer no embarazada.

Teniendo en cuenta las repercusiones de cualquier procedimiento quirúrgico durante la gestación, deseamos conocer la situación de pacientes sometidas a laparotomía durante la misma en el Servicio de Alto Riesgo. Teniendo en cuenta que constituye la primera indicación de cirugía y las pautas de manejo se dirigen hacia los siguientes parámetros:

- Hospitalización en Alto Riesgo y normas generales de manejo.

- Confirmar diagnóstico exacto de edad gestacional, con localización y dimensiones de la lesión, por ultrasonido.

- Establecer condiciones maternas que determinen conducta urgente. Manejo expectante si no existe, o la gestación es menor de 12 semanas.

- En gestaciones mayores de 12 semanas con sintomatología dolorosa, fenómenos de compresión, aumento de dimensiones de la lesión, o hallazgo ecográfico de la misma, se debe programar la laparotomía con anestesia peridural, biopsia por congelación, incisiones amplias, reposo estricto postcirugía, mínimo de 48 horas y en embarazos mayores de 24 semanas, un goteo con uteroinhibidores por lo menos durante 24 horas. En casos de neoplasia maligna conductas establecidas por el servicio de oncología de la institución.

- Al darlas de alta deben continuar en prenatal y se espera embarazo a término y parto por vía vaginal.

Con los resultados obtenidos se espera evaluar la conducta quirúrgica en gestaciones complicadas por masas ováricas sintomáticas.

Propósito. Evaluar la conducta quirúrgica en afecciones ováricas y embarazo, en el Servicio de Alto Riesgo, bajo diferentes aspectos.

Objetivo General. Presentar la experiencia institucional y demostrar el beneficio de la conducta quirúrgica durante la gestación.

Objetivos específicos.

1. Llevar a cabo la revisión de historias clínicas en todas las pacientes con diagnóstico de tumor de ovario y embarazo atendidas en el Servicio de Alto Riesgo.

2. Conocer la frecuencia, factores relacionados como edad, paridad y edad gestacional.

3. Establecer parámetros para el diagnóstico, ecografía y condiciones maternas al ingreso.

4. Determinar indicaciones para la laparotomía, tiempo de la gestación y manejo pre y postoperatorio.
5. Conocer la frecuencia, localización y tipo de la lesión correlacionada con anatomía patológica.

6. Conocer morbi-mortalidad del procedimiento, evolución posterior de la gestación, momento y vía del parto y condiciones de los recién nacidos. Además de morbi y mortalidad materna y perinatal.

7. Establecer los promedios de estadía.

8. Identificar para cada paciente las variables antes mencionadas.

\section{Materiales y métodos}

Tipo de estudio. La presente investigación corresponde a un estudio descriptivo con una corte transversal y una sola medición en una muestra de la población.

\section{Población de estudio}

1. Ubicación geográfica. Pacientes atendidas en el Servicio de Alto Riesgo del Instituto Materno Infantil.

2. Universo. 6.500 pacientes obstétricas atendidas desde el 1o. de enero de 1986 al 31 de octubre de 1993.

3. Muestra. 18 gestantes atendidas en el servicio en el mismo período..

\section{Recolección de la información}

1. Técnica de recolección. Mediante la revisión de historias clínicas y archivo privado de Alto Riesgo.

2. Procesamiento de la información. Los datos se tabularán en un computador PC 386.

3. Análisis de la información. Se utilizará análisis descriptivo a través de distribución de frecuencia, porcentajes, promedios e índices.

4. Presentación de resultados. En forma narrativa, cuadros y gráficas.

\section{Resultados}

\section{Movimiento del Servicio de Alto Riesgo}

Durante el período comprendido entre el 10. de enero de 1986 , fecha en la cual se inició la reorganización del Servicio de Alto Riesgo anteparto, hasta el 31 de octubre de 1993 se han atendido aproximadamente 6.500 embarazadas, las cuales corresponden al $7.4 \%$ de los ingresos al Instituto Materno Infantil. Con un promedio de atención de 100 pacientes nuevas por mes, 600 partos al año, (26\% pretérminos), una frecuencia de cesáreas del $37.6 \%$ y de abortos del $17.6 \%$. Cuadro No. 1.

\section{Cuadro No. 1 \\ MOVIMIENTO SERVICIO DE ALTO RIESGO}

$-7.4 \%$ de los ingresos al I.M.I.

- Atiende 100 pacientes nuevas por mes

- 600 Partos anuales

- Frecuencia de cesáreas del $37.6 \%$

- Frecuencia de abortos del $17.6 \%$

- Morbilidad materna 5.8\%

- Mortalidad materna 3.4 x 1.000

- Mortalidad perinatal del $52 \times 1.000$

- Porcentaje de ocupación $89.5 \%$

- Promedios de estadía 5.8 días. 
El $84 \%$ de los ingresos se relacionan con complicaciones propias de la gestación: amenaza de parto pretérmino, embarazo prolongado, cesárea anterior, hipertensión gravídica, hemorragias, embarazo múltiple, RPM. El 14\% representa las complicaciones médicas: infecciones de vías urinarias, síndromes convulsivos, diabetes, cardiopatías. El 2\% restante corresponde a complicaciones quirúrgicas: tumores torcidos de ovario, trauma, apendicitis. Con un índice general de morbilidad materna del $5.6 \%$, debido en primera instancia a la Endometritis Postparto (embarazo prolongado, hipertensión, hemorragias) y en cuanto a mortalidad materna un índice de 3.4 por 1.000 representado por causas no obstétricas: cáncer, tromboembolismo pulmonar. Cuadro No. 2.

\section{Cuadro No. 2}

\section{CAUSAS DE INGRESO AL SERVICIO}

$\begin{array}{lll}\text { - Complicaciones de la gestación } & : & 84 \% \\ \text { - Complicaciones médicas } & : & 14 \% \\ \text { - Complicaciones quirúrgicas } & : & 2 \%\end{array}$

La mortalidad perinatal depurada a un 52 por 1.000 con el pretérmino ocupando los primeros lugares tanto en la anteparto como en la neonatal precoz. Los promedios de estadía son de 5.8 días, con un porcentaje de ocupación del $89.5 \%$ para 24 camas disponibles.

\section{Frecuencia}

Teniendo en cuenta el número total de gestantes atendidas en Alto Riesgo, encontramos una frecuencia de 2.7 por $1.000,18$ gestantes, a quienes se les realizó laparotomía por patología ovárica sintomática. Lo anterior nos representa 3 pacientes nuevas por año. En relación con las complicaciones quirúrgicas del embarazo ocupan el primer lugar con motivo de ingreso al Servicio. Cuadro No. 3.

\section{Cuadro No. 3 \\ CIRUGIA POR AFECCIONES OVARICAS DURANTE LA GESTACION}

- Primera causa de ingreso en el grupo de complicaciones quirúrgicas.

- Tres pacientes anuales.

$-2.7 \times 1.000 .18$ gestantes.

\section{Factores relacionados}

En cuanto a edad, paridad y edad gestacional presentamos los siguientes promedios; edad 23.7 años (mínimo 14 máximo 34); paridad 2.3 (mínimo 1 máximo 6) y consultan hacia las 17.5 semanas de gestación (mínimo hacia las 8 y máximo hacia las 34 semanas). Cuadro No. 4.

\section{Cuadro No. 4 \\ CIRUGIA POR AFECCIONES OVARICAS DURANTE LA GESTACION}

\section{FACTORES RELACIONADOS}

$$
\begin{aligned}
& \text { - Edad } \\
& \text { - Paridad } \\
& \text { - Edad gestacional }
\end{aligned}
$$

\section{Motivo de consulta}

El $61.2 \%$ (11 pacientes) ingresaron por dolor persistente sobre las fosas ilíacas. En algunas con imposibilidad para la marcha y otros síntomas relacionados con fenómenos mecánicos de compresión.

En el 38.8\% restante (7 pacientes) el diagnóstico se hizo a través de hallazgo ecográfico, en estudio de rutina y lógicamente con gestante asintomática. Gráfica No. 1.

\section{Gráfica 1}

\section{CIRUGIA POR AFECCIONES OVARICAS MO'TIVO DE CONSULTA}

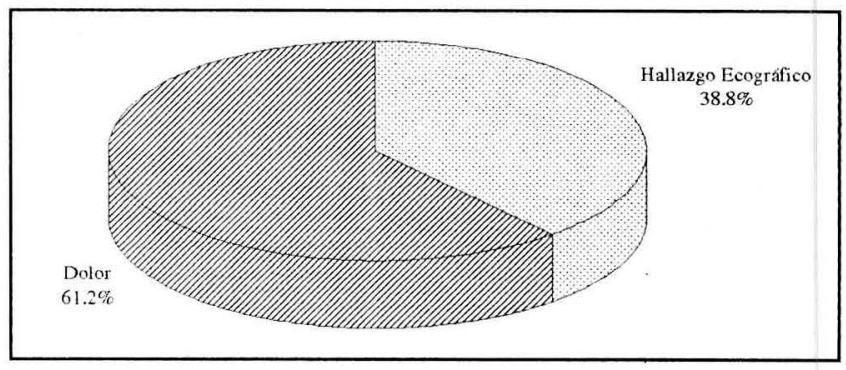

\section{Manejo y momento de la cirugía}

Una vez establecido el diagnóstico y determinada la no urgencia quirúrgica, se hizo manejo expectante en las gestaciones menores de 14 semanas. Además de las normas generales de seguimiento en paciente hospitalizada, el control ecográfico fue más estricto, con miras a determinar modificaciones en el tamaño de la lesión.

El tiempo de espera sin urgencia fue en promedio de 2 semanas; partiendo de la base y como veremos seguidamente que en gestaciones mayores de 14 semanas y con indicación de intervención no se hizo expectancia.

Las laparotomías se llevaron a cabo hacia las 18.8 semanas con un mínimo hacia las 12 y máximo hacia las 34 semanas.

Intervenciones en gestaciones avanzadas, después de las 22 hasta las 34 semanas se realizaron por condiciones maternas que suponían patología maligna. Cuadro No. 5 .

\section{Indicaciones de la cirugía}

La sintomatología dolorosa persistente determinó la programación de la cirugía en 13 gestantes, lo cual corresponde a un $72.2 \%$. Solamente 2 pacientes $(11.2 \%)$ fueron intervenidas de urgencia por cuadro de abdomen agudo y en los hallazgos se confirmó torsión de los quistes. 


\section{Cuadro No. 5 \\ CIRUGIA POR AFECCIONES OVARICAS DURANTE LA GESTACION}

\begin{tabular}{|c|c|}
\hline \multicolumn{2}{|c|}{ MANEJO Y MOMENTO DE LA CIRUGIA } \\
\hline - Expectante & $\begin{array}{l}\text { Menores de } 14 \\
\text { semanas. }\end{array}$ \\
\hline - Tiempo de espera & : 2 semanas. \\
\hline - Mayores de 14 semanas & : Laparotomía. \\
\hline - Edad gestacional & $\begin{array}{l}18.8 \text { semanas } \\
\text { (Min. } 12 \text {; máx. } 34)\end{array}$ \\
\hline
\end{tabular}

En un $27.7 \%, 5$ embarazadas, con gestaciones mayores de 20 semanas, el seguimiento ecográfico mostró aumento de las dimensiones de la lesión inicial y según se mencionó anteriormente, en 2 de ellas con sospecha de malignidad. Gráfica No. 2.

\section{Gráfica 2}

\section{CIRUGIA POR AFECCIONES OVARICAS INDICACIONES DE LAPAROTOMIA}

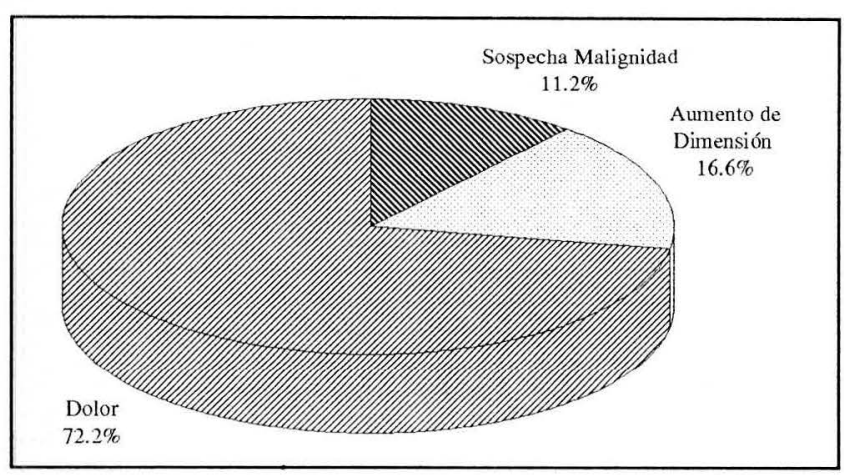

7. Condiciones para la cirugía-anestesia y complicaciones

Previa valoración pre-anestésica, reserva de sangre y solicitud de patólogo en sala para biopsia por congelación, todas las gestantes se intervinieron con anestesia peridural, incisión de laparotomía amplia y protección cuidadosa del útero grávido.

En el $94.5 \%$ se practicó la salpingooforectomía del anexo comprometido. Una gestante, $5.5 \%$ con sintomatología dolorosa, urinaria y de obstrucción, en la laparotomía se encontraron 2 miomas pediculados en la cara anterior y se practicó miomectomía.

En los casos que se confirmó malignidad solamente se extirpó la lesión inicial y el manejo subsiguiente de acuerdo a los protocolos del servicio de oncología de la institución.

En 2 pacientes se encontró torsión de la lesión y no se presentaron complicaciones anestésicas ni hemorrágicas, ni rupturas de las lesiones en el transoperatorio. Cuadro No. 6.

\section{Manejo postoperatorio}

No se utilizaron progestágenos en ninguna de las gestaciones. En el $83.3 \%$ (15 gestantes) se recomendó

\section{Cuadro No. 6 \\ CIRUGIA POR AFECCIONES OVARICAS DURANTE LA GESTACION}

\section{MANEJO INTRA-POSTOPERATORIO}

- Anestesia peridural.

- Incisión mediana infraumbilical amplia.

- Salpingooforectomía del anexo

comprometido

$94.5 \%$

- Miomectomía : $5.5 \%$

- Uteroinhibición profiláctica : $\quad 16.7 \%$.

sedación con prometacina y reposo en cama las primeras 48 horas.

Ordenamos goteos con uteroinhibidores del grupo de la terbutalina en 3 gestantes, $16.7 \%$ durante las primeras 24 horas del postoperatorio.

\section{Localización de la lesión y anatomía patológica}

La localización de la lesión en el lado derecho fue más frecuente que en el anexo izquierdo, con un $72.2 \%$ (13 casos) y $27.8 \%$ (5 casos) respectivamente.

El tamaño aproximado de las masas estuvo entre 8 y $24 \mathrm{~cm}$ con un promedio de $12 \mathrm{~cm}$.

Reportamos 16 resultados $(88.8 \%)$ compatibles con patología benigna y 2 pacientes $(11.2 \%)$ con cambios malignos. Gráfica No. 3.

Se distribuyeron así: para los benignos en orden de frecuencia cistadenoma seroso $27.8 \%$ (en la literatura se reporta un primer lugar para los dermoides); dermoide

\section{Gráfica 3}

CIRUGIA POR AFECCIONES OVARICAS LOCALIZACION Y TIPO DE LESION

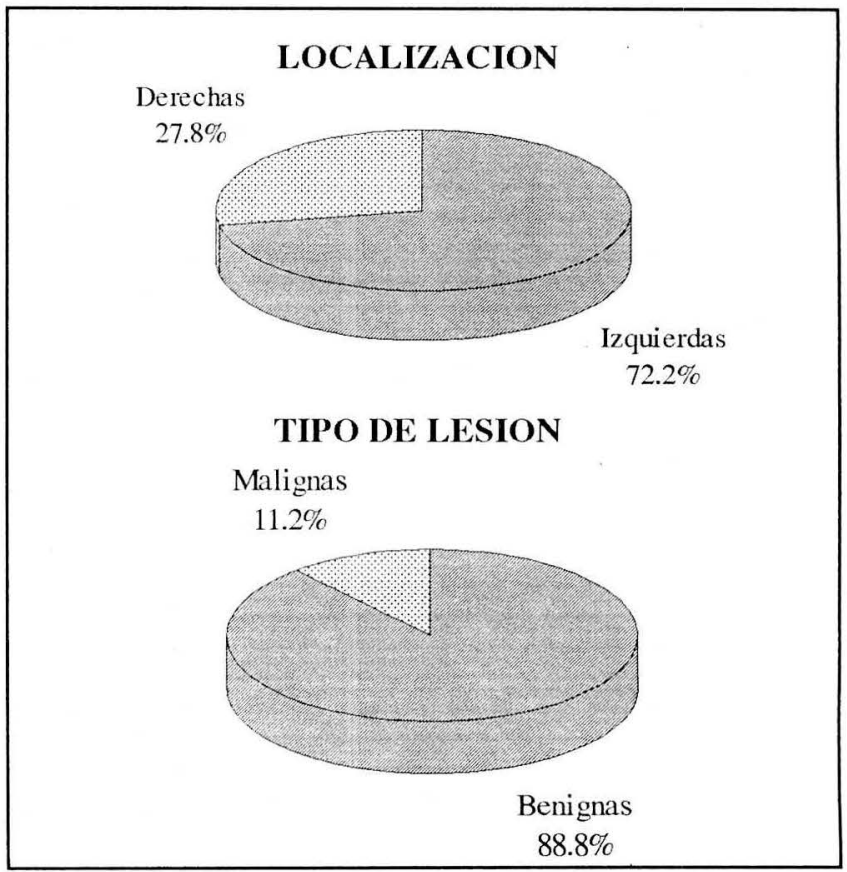


$22.3 \%$; quistes simples $16.7 \%$; endometrioma $5.5 \%$; fibroma ovario izquierdo $5.5 \%$; mioma uterino $5.5 \%$ y quiste simple de paraovario $5.5 \%$. Gráfica No. 4.

En cuanto a las neoplasias malignas, el $11.2 \%$ para esta serie sobrepasa las cifras reportadas en la literatura, las cuales varían entre el 2-5\%. Tenemos un Cistadenocarcinoma Seroso Papilar y un Tumor de Krukenberg.

\section{Gráfica 4 \\ CIRUGIA POR AFECCIONES OVARICAS PAATOLOGIA BENIGNA $88.8 \%$}

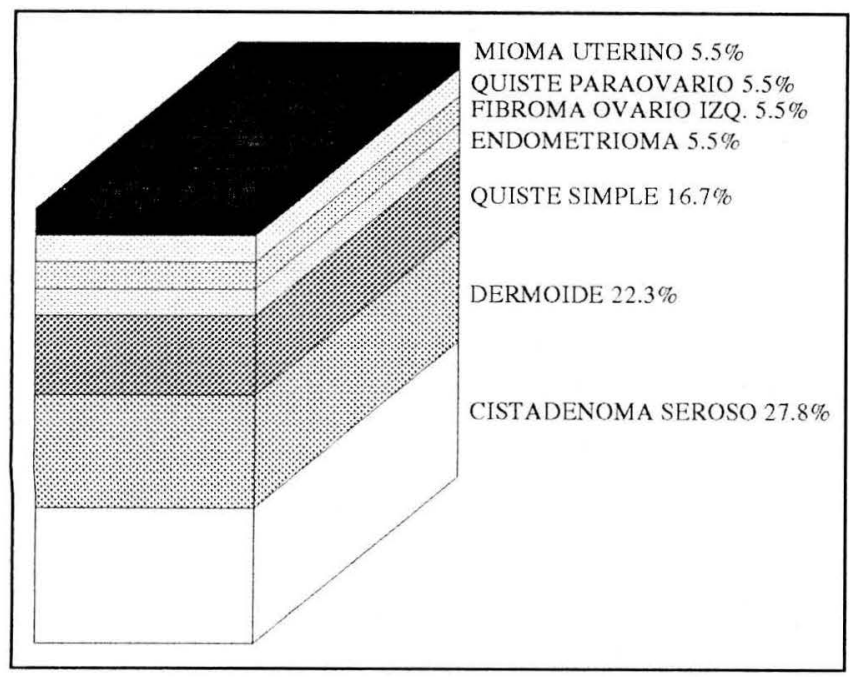

10. Momento y vía del parto. Indicaciones de Cesárea

Es grato comunicar el hecho de no haberse presentado amenazas de aborto, abortos o amenazas de parto pretérmino en los post-operatorios.

Solamente tenemos una gestación que terminó hacia las 32 semanas, pero por muerte materna debido a una carcinomatosis.

El promedio del parto es hacia las 38.3 semanas con un mínimo de 32 y un máximo de 41 semanas.

Con parto por vía vaginal espontáneo se atendieron 13 gestantes $(81.2 \%)$; se incluye paciente con diagnóstico de cistadenocarcinoma seroso.

La operación cesárea se indicó en 3 embarazadas $(18.8 \%)$ por antecedentes de miomectomía, una crisis hipertensiva y un trabajo de parto prolongado; es decir que el antecedente de la cirugía previa determinó la intervención solamente en un 5.5\%.

Aclaramos estos resultados, teniendo en cuenta 16 casos, pues una gestante se encuentra en prenatal al momento del análisis de estos resultados y el otro caso corresponde a la muerte materna por carcinomatosis. Cuadro No. 7, Gráfica No. 5.

\section{Condiciones de los recién nacidos}

Igualmente sobre 16 recién nacidos obtuvimos 9 de sexo masculino (56.2\%) y 7 de sexo femenino (43.8\%); con apgar promedio de $9 / 10$ (mínimo $6 / 10$ y máximo $10 /$ 10) y peso promedio de 2.998 gramos. (Mínimo 2.200 y máximo 4.000 gramos). Cuadro No. 8 .

\section{Cuadro No. 7 \\ CIRUGIA POR AFECCIONES OVARICAS DURANTE LA GESTACION}

\section{EVOLUCION OBSTETRICA}

- Amenaza de aborto

- Abortos

- Parto pretérmino
- Amenaza de pretérmino

No

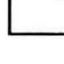

\section{Gráfica 5 \\ CIRUGIA POR AFECCIONES OVARICAS MOMENTO Y VIA DEL PARTO}

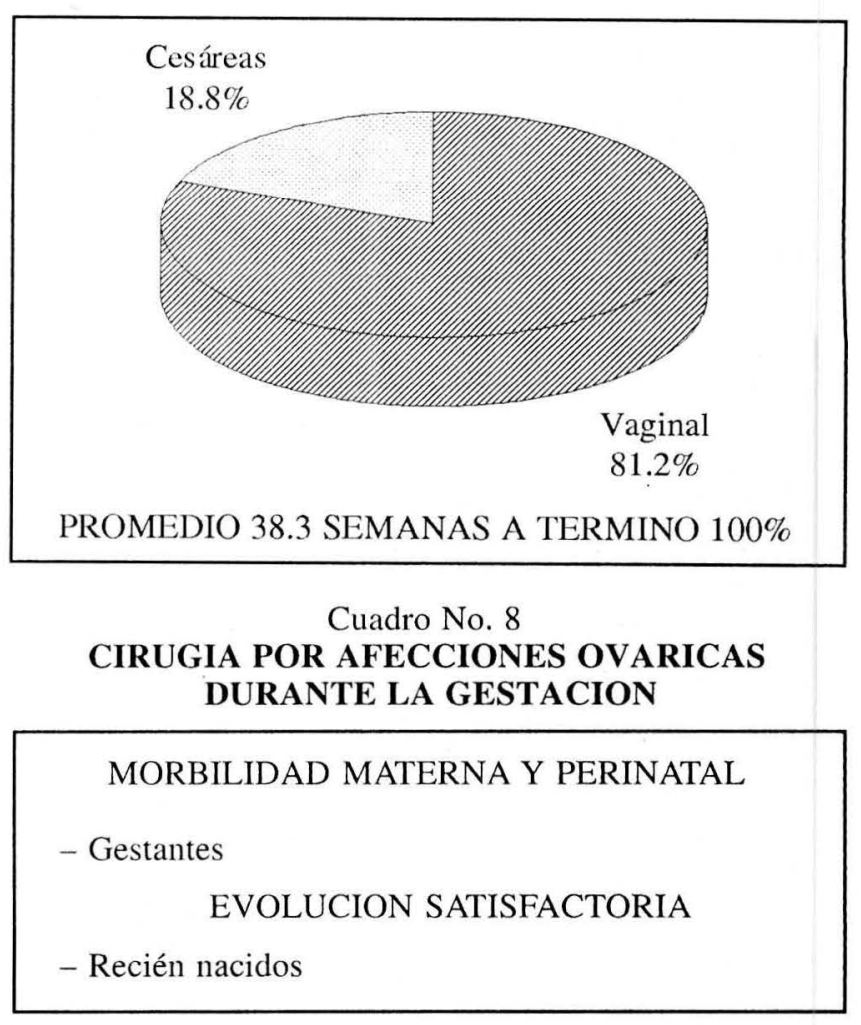

\section{Morbilidad materna}

No reportamos morbilidad materna relacionada con la entidad de base. Tal vez anotar una gestante con diabetes tipo II controlada metabólicamente con insulina y laparotomía a las 24 semanas sin complicaciones.

\section{Mortalidad materna}

Desafortunadamente presentamos un índice de mortalidad para esta serie de 62.5 por 1.000. Caso del Tumor de Krukenberg.

\section{Morbilidad perinatal}

Todos los recién nacidos evolucionaron satisfactoriamente. Se observó en pediatría el hijo de madre diabética de acuerdo con las normas establecidas por la clínica de diabetes y embarazo de la institución. 


\section{Mortalidad perinatal}

Anteparto y concomitante con el deceso materno por carcinomatosis, ocurrió el único caso de mortalidad perinatal, para un índice también de 62.5 por 1.000 Cuadro No. 9.

\section{Cuadro No. 9 \\ CIRUGIA POR AFECCIONES OVARICAS DURANTE LA GESTACION}

\section{MORTALIDAD MATERNA Y PERINATAL}

$$
\text { Indice : } 62.5 \times 1.000
$$

- Tumor de Krukenberg. Carcinomatosis

- Muerte fetal anteparto.

\section{Estadía}

El mínimo de días de hospitalización fue de 8, con un máximo de 45, para un promedio de 15.2 días (sobrepasa los promedios regulares del Servicio).

\section{Discusión de resultados}

1. Presentamos la experiencia institucional con un grupo de 18 pacientes a quienes se les practicó laparotomía con salpingooforectomía durante la gestación; debido a lesiones ováricas, las cuales de alguna forma estaban comprometiendo el curso normal del embarazo.

2. Reportamos los beneficios del procedimiento, con mejoría de la sintomatología inicial, sin compli- caciones intra ni postoperatorias se logra mantener la gestación hasta el término y con parto por vía vaginal en la mayoría.

3. Tenemos una frecuencia mayor de patología benigna con cistadenoma serosos ocupando el primer lugar; pero el número de pacientes con neoplasia maligna sobrepasa lo esperado o reportado en otros estudios.

4. La mortalidad materna y perinatal se relacionan con neoplasia maligna y no como secuelas de la intervención diagnóstica.

\section{Recomendaciones}

Continuar con manejo expectante antes de las 14 semanas con paciente hospitalizada. Después de este tiempo intervenir en el momento del diagnóstico independientemente del motivo de consulta (dolor o hallazgo ocasional). Cuadro No. 10.

\section{Cuadro No. 10 \\ CIRUGIA POR AFECCIONES OVARICAS DURANTE LA GESTACION}

\section{RECOMENDACIONES}

- Manejo expectante, si no hay urgencia, antes de las 14 semanas.

- Laparotomía después de las 14 semanas una vez confirmado el diagnóstico.

\section{BIBLIOGRAFIA}

1. Ballard Charles A. Ovarian Tumors Associated with Pregnancy Termination Patients. Am. J. Obstet. Gynecol. 1984; 149: 384-387.

2. Disaia Philip J. and Ceasaman Willian T editors. Ginecología oncológica. 3a. edición. Buenos Aires, 1991. Panamericana S.A.

3. Lavery J., Patrick Koontz., William L., Layman L et al. Sonographic evaluation of the adnexa during early pregnancy. Surg. Ginecol. Obstet. 1986; 163: 319-323.

4. Barber HRK and Graber EA Editors. Surgical disease in pregnancy, Philadelphia. 1978, WB Saunders Co.

5. Sundo Christian S., Keith Y., Kamemoto Lori E et al. Adnexal masses in Pregnancy: Ocurrence by ethnic Group. Obstet. Gynecol. 1990; 75 : $38-40$.

6. Kier R., McCarthy Shirley M., Scoutt Leslie M et al. Pelvic Masses in Pregnancy: MR Imaging Radiology. 1990; 176: 709-713.

7. Dantfoth DN Editor. Tratado de Obstetricia y Ginecología 4a edición. México D.F., 1988. Interamericana S.A.

8. Dessel T., Van Hameeteman TM and Wagenaar SJ. Mucinous Cystadenocarcinoma in Pregnancy. Caase report. Br. J. Obstet. Gynecol. 1988; 95: 527-529.

9. Manual de Normas de Manejo del Cáncer Genital Femenino. Instituto Nacional de Cancerología. Santafé de Bogotá, marzo 1993.

10. Koonings Paul P., Platt Lawrence D. and Wallace R. Incidental Adnexal Neoplams at Cesarean Section. Obstetric Gynecol. 1988; 72 : 767-769.

11. Roberts James A. Management of Gynecologic Tumors during Pregnancy. Clin. Perinatol., 1983; 10: 369-382.

12. Guzmán DR. Cáncer y Embarazo. Instituto Materno Infantil. Santafé de Bogotá, febrero 1988.
13. Vanslooten Andrew J., Rechener Stephen F., Dodds William G. Recurrent Maternal Virilization during Pregnancy Caused by Bening Androgen-producing Ovarian Lesions. Am. J. Obstet. Gynecol. 1992; 167: 1342-1343.

14. McGowan L. Enfermedades quirúrgicas del Ovario en el Embarazo. Clínicas de Perinatología. 1983; 10: 1029-1043.

15. Creasy Robert K and Resnik R. editors. Maternal-Fetal Medicine Principles and Practice. Second Edition. Philadelphia. 1989, WB Saunders Co.

16. Piver M., Steven B., Trudy R., Piedmonte Marion et al. Epidemiology and Etiology of Ovarian Cancer. Sem Oncol. 1991; 18: 177-185.

17. Olt George B., Andrew and Bast., Robert C. The role of Tumor Markers in Gynecology Oncology. Obstet. Gynecol. Survey. 1990; 45: 570-577.

18. Friedlander Michael L. and Dembo., Alon J. Pronostic Factors in Ovarian Cancer. Sem. Oncol. 1991; 18: 205-212.

19. Principios y Normas Generales de Ginecología. Universidad Nacional de Colombia. Facultad de Medicina. Departamento de Ginecología y Obstetricia. Santafé de Bogotá, 1989.

20. Kung Annie WC., MA John TC., Wang Christina and Young., Rosie T. Hyperthyroidism during Pregnancy due to coexistence of Struma Ovaril and Graves Disease. Postgrad Med J. 1990; 66: 132-133.

21. Van Der Zee AGJ., Bruijin HWA., Bouma J et al. Endodermal Sinus Tumor of the Ovary during Pregnancy: A case report. Am. J. Obstet. Gynecol. 1991; 164: 504. 506.

22. Kim Doo Sang., Moon Hyung., Lee Jar A et al. Anticancer Drugs during Pregnancy. Are we able to discard them? Letter Am. J. Obstet. Gynecol. 1992; 166: 265-266. 